\title{
Combining similarity functions and majority rules for multi-building, multi-floor, WiFi Positioning
}

\author{
Nelson Marques, Filipe Meneses and Adriano Moreira \\ Mobile and Ubiquitous Systems research group \\ Centro Algoritmi, University of Minho \\ Guimarães, Portugal \\ \{nelson.marques, filipe.meneses, adriano.moreira\} @algoritmi.uminho.pt
}

\begin{abstract}
Fingerprint is one of the most widely used methods for locating devices in indoor wireless environments and we have witnessed the emergence of several positioning systems aimed for indoor environments based on this approach. However, additional efforts are required in order to improve the performance of these systems so that applications that are highly dependent on user location can provide better services to its users. In this work we discuss some improvements to the positioning accuracy of the fingerprint-based systems. Our algorithm ranks the information about the location in a hierarchical way by identifying the building, the floor, the room and the geometric position. The proposed fingerprint method uses a previously stored map of the signal strength at several positions and determines the position using similarity functions and majority rules. In particular, we compare different similarity functions to understand their impact on the accuracy of the positioning system. The experimental results confirm the possibility of correctly determining the building, the floor and the room where the persons or the objects are at with high rates, and with an average error around 3 meters. Moreover, detailed statistics about the errors are provided, showing that the average error metric, often used by many authors, hides many aspects on the system performance.
\end{abstract}

Keywords - fingerprinting; indoor positioning; rssi; wlan; mobile computing)

\section{INTRODUCTION}

Location-based services (LBS) are one of the most popular classes of mobile services. Finding the position of a person or an object in the real world is a feature that has emerged in many mobile computing applications. Location-based services have a tremendous impact on our daily lives. Such services are especially available in outdoor spaces, typically using the Global Positioning System (GPS) for positioning. Despite an acceptable level of accuracy and precision, GPS systems require line of sight access to the orbiting GPS satellites. Furthermore, much of our lives are spent in indoor spaces, where the GPS signal is seriously compromised. Thus positioning techniques for indoor environments are becoming a market segment that provides new business opportunities.

The trust, the simplicity and the accuracy are some of the key factors in LBS [1]. In particular, a positioning system for indoor environments opens the door to a range of services targeted to spaces such as offices, shopping malls and transport infrastructures like airports and subway stations. One good example of indoor LBS is the location of doctors in a hospital environment where, in critical cases, doctors should be located and notified quickly.

\section{A. WLAN Fingerprinting}

Different wireless technologies can be used for supporting wireless indoor positioning systems. However, the constant development and the increasing popularity of IEEE 802.11 wireless local area networks (WLAN) lead to the development of many positioning systems that exploit these infrastructures. Such networks have their main benefit in the fact that they are standardized and they carry a high level of acceptance. Moreover, nowadays, the WiFi interface is the most common network interface found in mobile devices for data transfer and Internet access. The large coverage and the high-speed transmission rate are other features, which make WiFi to be recognized as a good infrastructure network to support a positioning system for the indoor environment.

Most of WLAN-based positioning systems in indoor environments are based on the Received Signal Strength Indicator (RSSI) value. In these positioning methods there are two phases: calibration and operation. In the calibration phase, which occurs before the operational phase, a radio map of the area where the mobile devices are to be detected is constructed. Later, during the operational phase, a user (or a device) obtains the signal strength of all visible WLAN access points and creates a sample that is sent to the server to be compared with the samples on the radio map. Consequently, the user's position corresponds to the position associated with the most similar sample in the radio map.

The accuracy and the cost of computing are influential factors when choosing the technique for a positioning system. One of the major advantages of the fingerprinting-based methods is that they do not require the installation of any additional hardware. If a WLAN infrastructure exists, the position of the user can be obtained without additional hardware installation, thus the costs are smaller and the service can be offered in a shorter time.

However, WLANs were not natively designed to support a positioning function. Taking into account the existing obstacles introduced by the indoor environment, including reflections and multipath interference, the spread of radio signal in indoor 
environments is very hard to predict. Typically, in WLANbased positioning systems, the user carries the mobile device with him. The effect of the user's presence close to the antenna is also an influential factor in the measured RSSI values [2]. For these reasons, the basic fingerprinting technique is often combined with other techniques, such as Inertial Measurement Units or RFID, in order to overcome the outliers typical of WiFi fingerprinting and to improve accuracy. However, hybrid solutions demand the use of specific tags and the installation of additional hardware. In the work described in this paper, we base our solution on fingerprinting only in order to enable the positioning of off-the-shelf devices (tags, laptops, tablets and smartphones).

\section{B. Issues in Fingerprinting}

The signal spread in indoor spaces is extremely complex and signal strength changes very often and fast. The performance of the positioning system is often degraded because of the variability of the RSSI. In general, the received signal is composed of a Line Of Sight (LOS) component and several reflected signals with different levels of attenuation. Moreover, at a fixed location, the RSSI value from an AP varies throughout the time. This effect is caused by changes in the environment such as close/open doors, variations on the weather conditions like humidity and temperature, or by people moving around [3]. In addition, an indoor space includes walls, floorboards and doors, which tend to block or reflect radio signals in complex ways. This variation is mainly due to the effect of multipath. Inevitably, the problem of multipath has a significant effect when the positioning system is implemented in a real environment. Traditionally, the variation of the RSSI is generalized as an additional noise signal. In order to minimize that noise, filters are normally applied [6].

Fig. 1 shows an example of the RSSI variations along the time. The samples were taken over a period of 10 hours, with a five seconds interval, starting at midnight and ending at $10 \mathrm{am}$. Two collecting setups have been used, using similar USB WiFi network adapters (same brand and model), but connected to two different computers with different operating systems (Windows XP Server and Windows Seven). The USB adapters were placed within a few centimeters from each other. Each one of the lines in Fig. 1 shows how the signal level from one specific AP oscillated along the time.

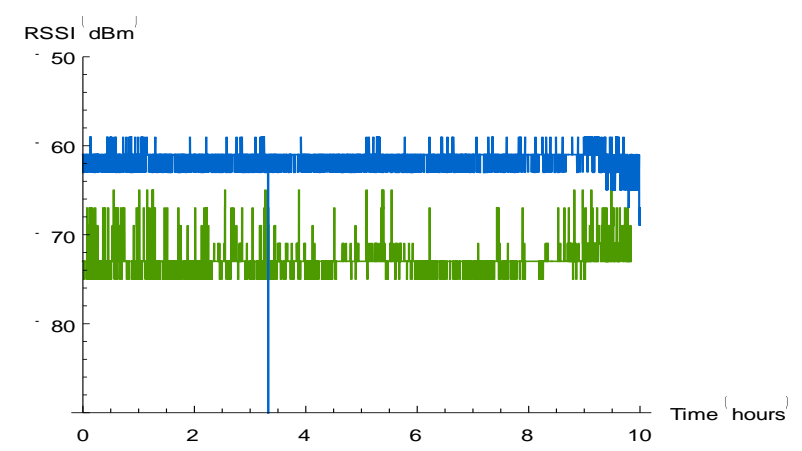

Figure 1. Variation on the RSSI value of a single AP, for two different collecting setups, over a period of 10 hours.
These results are a bit surprising, and illustrate two important aspects on the variability of the WiFi signals. The first is related to the collecting setup: the use of two different operating systems, and consequently two different device drivers, has a significant impact on the absolute values of the measured RSSI and also on its variation span. One possible cause for these differences, or part of them, could be differences on the hardware of the used USB network adapters. However, similar results were obtained with alternative hardware. Other causes for the observed variations might include the power source used to power the USB devices or other factors that we are still trying to investigate.

The second aspect, clearly visible in this example, is that the presence of people in the space deeply affects the measured RSSI values. This is visible to the right of $8 \mathrm{~h} 00$, which corresponds to the time people start arriving to work. However, this effect is much more visible in one of the setups (XP Server) than on the other one, where the measured RSSI values are a lot more stable. On the other hand, during the night, the stability of the RSSI values is clearly higher for the XP Server setup.

The heterogeneity of devices (WiFi network adapters) can also reduce the performance of the positioning system [4]. In fingerprinting, RSSI values stored in a radio map are compared with values obtained by mobile devices. Due to the differences in hardware and software (device drivers) between different devices, the RSSI values obtained by the devices can be different from values stored in the radio map, and this may degrade the positioning accuracy [6].

Different devices not only get different signal strengths, but also detect a different set of APs. One device can continuously detect a nearby AP, while another device in the same position may never be able to detect that same AP. Fig. 2 shows another example on how the collecting setup might impact the stability of the RSSI values. Fig. $2 a$ and $2 b$ shows the number of APs detected by the two above described collecting setups, over the same period of time (from midnight to $10 \mathrm{am}$ ). Fig. 2c shows the corresponding distributions. Again, these results illustrate the different levels of variability due to the used of two different software configurations, even when using the same hardware (same brand and model of network interface). However, the impact of the presence of people, after the early morning, is not so clearly visible.

In fingerprinting-based positioning systems, the size of the radio map is an influential factor to the computational load in the operational phase [7]. A positioning system for a relatively large area incurs in a high computational complexity. On the other hand, the accuracy of the positioning system strongly depends on the number of calibration points and on the quality of the samples in the radio map. A more refined radio map, with more calibration points, can considerably increase the accuracy of the positioning system [1]. However, a radio map with more calibration points makes the operational phase computationally more complex and more time-consuming. Nevertheless, especially in places with significant size, a part of the samples obtained during the calibration phase is not relevant to the operational phase. 


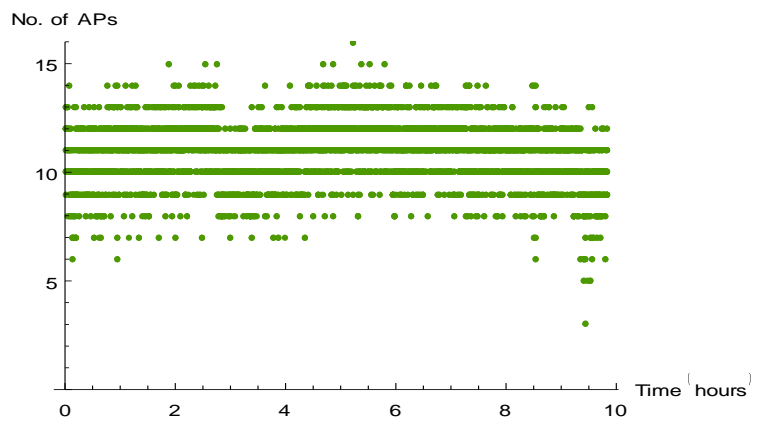

(a)

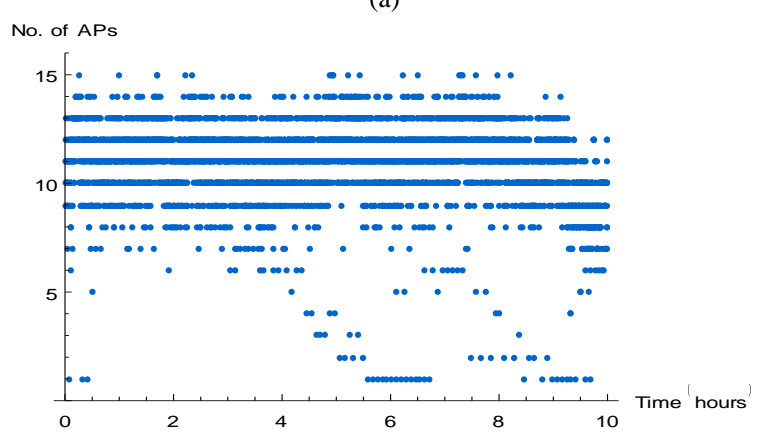

(b)

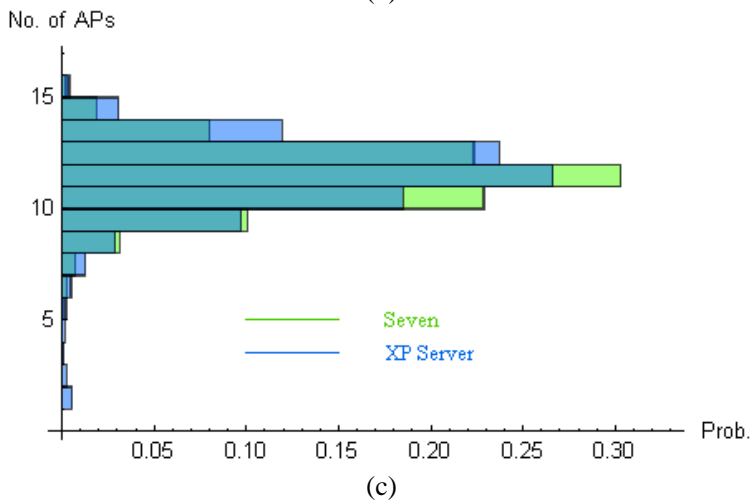

Figure 2. Number of observed APs: (a) Windows Seven setup; (b) Windows XP Server setup; (c) Probability distribution.

For all these reasons, the optimization of the radio map has become a challenge in the fingerprinting-based positioning systems in order to fulfill the requirements of real-time applications.

\section{Performance Evaluation}

Traditionally indoor positioning systems provide only the geometric position of the user. However, there are many applications that can benefit of other location information such as the building, floor or the room where users are located.

One of the goals of this research is to show a new positioning algorithm to solve the problem of positioning in a multi-building, multi-floor and multi-room environment. The proposed method starts by estimating the building where the user is located, then determine the floor, the room and, finally, the most probable geometric position within the room. The results demonstrate that our strategy is feasible with a very acceptable accuracy. The second objective is to study the impact of different similarity functions on the accuracy of the positioning system. The impact of the adopted strategy on the computational load is also analyzed.

In addition, there are many authors that present the results of their systems, which are very interesting, although they do not reflect the real accuracy. Therefore, it is also one of the objectives of this research to provide a new set of metrics to evaluate the performance of positioning systems. The metrics include the average error, the maximum geometric error, the error rate on the estimation of the right building, floor and room, and the time required to compute each estimate. Moreover, through the results we will show the error distribution in a very simple way.

The rest of the paper is organized as follows. The next section presents our positioning method where we show how our system builds the radio map and how it is compressed efficiently. In section III we describe the proposed algorithm. In section IV we present the real experiments. The results and the discussion are presented in Section V. Finally, in the last section, some conclusions are extracted from the work done.

\section{RADIO MAP}

The construction of the radio map starts with the selection of several calibration points with the assistance of a building plan. The RSSI values associated with each one of the APs are collected at the calibration points over a certain period of time and stored in the radio map.

The $i$ th element in the radio map has the following form:

$$
M_{i}=\left(B_{i}, H_{j}, \vec{a}_{i, k}, \emptyset_{i}\right), i=1 \ldots M, j \in\{80,160,240\}, k \in N_{k}
$$

where $B_{i}$ corresponds to the $i$ th calibration position and $H_{j}$ to the height of measurement (three height are considered, at 80, 160 and $240 \mathrm{~cm}$ from the floor level, to enable 3D geometric positioning). The vector $a_{i, k}$ represents the RSSI values measured from the access point $k$ at position $B_{i}$. The parameter $\phi_{i}$ contains any additional information necessary to estimate the location in the operational phase, such as the identification of the building, floor and room.

The radio map is modified or pre-processed before the operational phase (as depicted in Fig. 3). The motivation for the pre-processing is to reduce the memory requirements and reduce the computational cost of estimating the location.

\section{A. Filtering the calibration samples}

Filtering can be used to remove data that is not useful for the estimation process, or that might contribute to a degraded accuracy. In our case we are filtering out any RSSI values that are not related to well-known APs (e.g. mobile hotspots) or that are related to distant APs deployed in other buildings.

\section{B. Normalizing the calibration samples}

The normalization process creates calibration samples that include all the observed APs, in all samples, and assigns a default values to the RSSI of the APs that were not visible in each sample. The order of the APs in each sample is also made uniform to facilitate the computational process during the operational phase. 


\section{Final radio map}

Once the radio map is constructed, the person or object may be located during the operational phase by comparing its observed radio signals (sample) to the radio map stored entries. However, the radio map contains a large number of entries. Matching the sample collected by the WiFi terminal with all the entries in the radio map requires too much time. This is even more evident when dealing with large areas involving many buildings as the radio map may contain many thousands of calibration samples.

Many authors proposed several approaches aiming to compress the radio database. According to [8], some techniques such as Principal Component Analysis (PCA) and Kernel Canonical Correlation Analysis (KCCA) have been proposed to compress the radio map. In addition, an alternative may be to reduce the number of measurements (i.e., reduce the density of the radio map).

However, a more refined radio map, with more calibration points, can considerably increase the accuracy of the positioning system [1]. For that reason, and because the 3D position of the target devices in the operational phase is unknown, our radio map takes into account the height where the measurements are taken. At each calibration point three measurements are performed at three different heights. In our tests the calibration samples were obtained at 80, 160 and 240 $\mathrm{cm}$ from the floor level.

Computing the similarity of the test sample with all the calibration samples in the radio map is a very time consumption task. In order to minimize the processing time, we propose a solution where the radio map is first filtered, for each test sample, based on a very simple and computational efficient process. This process is described in the next section.

\section{POSITIONING AlgORITHM}

The main purpose of the operational phase is to estimate the position of a person or an object from the received fingerprint, which includes RSSI measurements from all visible access points. In our case, up to three measurements are taken, with a one second interval, at the same location before calculating the position. The average of these measurements is used as the test sample. Fig. 4 illustrates the proposed architecture for the localization phase to estimate the position of mobile terminals.

Our algorithm is similar to the positioning mechanisms suggested by several authors. The assumption behind these mechanisms is that at a certain point, a user can detect the signal from different APs. This collection of APs and the respective RSSI values represent a fingerprint that is unique to each position. Our method uses the basic algorithm for fingerprinting but with an improvement aiming to reduce the processing time. The estimation process is based on a sequence of filtering functions and majority rules. At each step, we estimate the most probable building, then the most probable floor, the most probable room and, finally, the geometric position within the room. In this process, computing the similarity of the test sample with the samples in the radio map is done only after the estimation of the most probable building, thus reducing considerable the required number of operations and processing time.

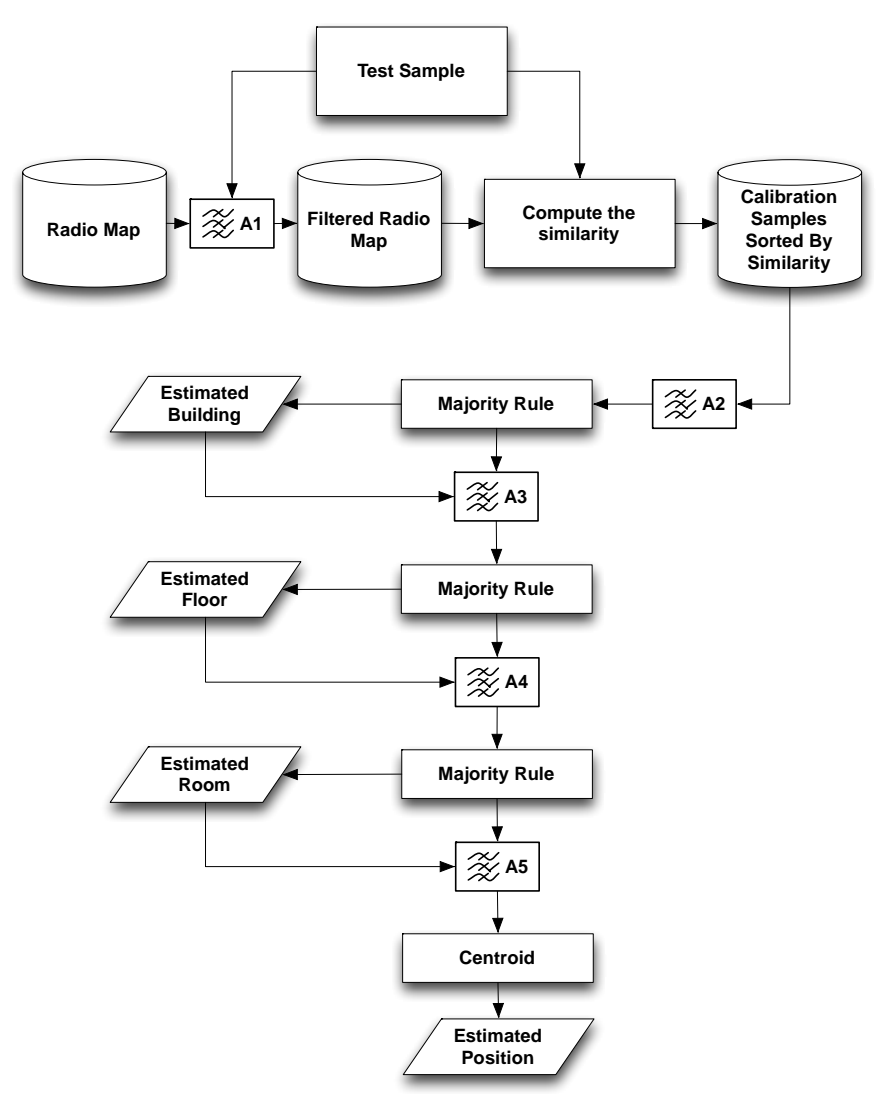

Figure 4. Architecture of the position estimation process.
Figure 3. A schematic overview of the radio map construction.

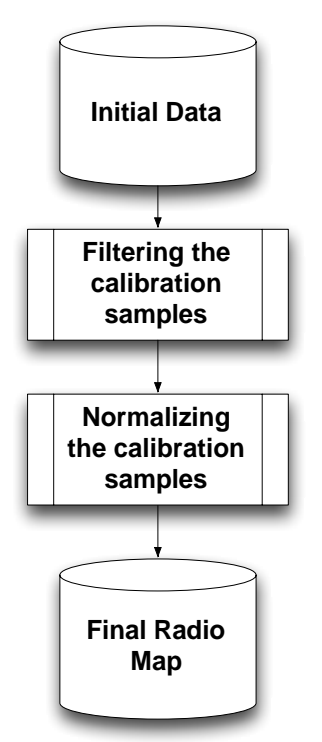


In the operational phase, the mobile device of a user obtains the list of APs and the respective intensities of the received signal at a given location (Test Sample). We begin the positioning process with the construction of a Filtered Radio Map where the Test Sample is used as a key. The aim of the filtering function is to reduce the computational cost of the following step, where the similarity between the Test Sample and each one of the Calibration Samples in the Filtered Radio Map is computed. The following steps are based on filtering stages and majority rules. At the end of each one of these steps, we get the estimated building, floor and room. Finally, the estimated geometric position is obtained by computing the centroid of the most similar Calibration Samples from the estimated room.

\section{A. Radio Map Filtering}

The rationale behind the initial filtering of the radio map is that the position where a Test Sample is taken is dominated, in terms of radio signal level, by one or two APs. Therefore, all the Calibration Samples where the strongest AP is not the same as the strongest AP in the Test Sample are not relevant. However, the fluctuations on the signal level must be taken into account.

By taking into account the two access points with better RSSI values in the Test Sample, the Filtered Radio Map is constructed taking into account two conditions:

- If the difference between RSSI values of the two strongest APs in the Test Sample is larger than a predefined threshold maxDiff (meaning that one of the APs dominates), then include in the Filtered Radio Map all the Calibration Samples where the strongest $\mathrm{AP}$ is the same as the strongest AP in the test sample;

- Otherwise, include in the Filtered Radio Map all the Calibration Samples where the strongest AP in the Test Sample is one of the two strongest in the Calibration Samples.

If the Test Sample is normalized as the Calibration Samples were in the calibration phase (section II.B), the filtering process can be computationally very efficient.

The block named A1 in Fig. 4 represents this filtering stage of the positioning estimation process.

\section{B. Similarity Functions}

Given a Test Sample and a Filtered Radio Map, the estimation process proceeds with the computation of the similarity between the Test Sample and each one of the Calibration Samples in the Filtered Radio Map. Computing the set of similarities is the most time consuming task in the estimation process. Therefore, minimizing the number of calibration samples on the filtered radio map contributes to minimize the overall estimation time.

The choice of the similarity function has also a considerable impact on the accuracy of the positioning solution. Some similarity functions perform better than others, but they also require different levels of computational effort. Next we describe some of the alternatives for the similarity function. In the following sections we assess the performance of those similarity functions, both from the point of view of accuracy and computational effort.

The similarity between two samples can be seen as a distance in a multi-dimensional space. We define the similarity $S$ between two samples $s_{1}$ and $s_{2}$ as:

$$
S\left(s_{1}, s_{2}\right)=D\left(s_{1}, s_{2}\right)-\alpha \times n C M+\beta \times n N C M
$$

where $D\left(s_{1}, s_{2}\right)$ is the distance function to use, $n C M$ is the number of common MACs (APs) present in both samples, $n N C M$ is the number of non-common MACs, and $\alpha$ and $\beta$ are weighting parameters. The lower the value of $S$, the more similar are the samples.

\section{1) Euclidean distance}

One of the most commonly used metrics to calculate the signal distance between two samples is the Euclidean distance. The Euclidean distance is defined by:

$$
D\left(s_{1}, s_{2}\right)=\sqrt{\sum_{i=1}^{n}\left(s_{1, i}-s_{2, i}\right)^{2}}
$$

where $n$ is the number of common MAC addresses present in both radio samples, and $s_{x, i}$ is the RSSI value of AP $i$ in sample $x$. This function considers all APs that are observed in both samples.

\section{2) Manhattan distance}

The Manhattan distance uses the sum of the absolute differences rather than their squares, and is defined by:

$$
D\left(s_{1}, s_{2}\right)=\sum_{i=1}^{n}\left|s_{1, i}-s_{2, i}\right|
$$

\section{3) Tanimoto distance}

The Tanimoto metric normalizes the difference, with -1 for maximum similarity and 0 for minimum similarity. The Tanimoto distance is given by:

$$
D\left(s_{1}, s_{2}\right)=\sum_{i=1}^{n} \frac{s_{1, i} \cdot s_{2, i}}{s_{1, i}^{2}+s_{2, i}^{2}-s_{1, i} \cdot s_{2, i}}
$$

Each one of the above described functions can be used in the estimation process, with different results. These results are described in section V-B.

\section{Filtering and Majority Rules}

Given a list of calibrated samples sorted by similarity, we propose an estimation process based on a sequence of filtering and majority rules steps. At each step, one dimension of the estimated location is obtained: building, floor, room, and geometric position.

To estimate the most probable building, one selects the $L 1$ most similar calibration samples (filtering step A2) and, from those, compute the most frequent building using a majority rule (the most frequent building within the $L 1$ samples is selected as the estimated building). The output of this step is an estimation 
of the right building and a set of $L 2 \leq L 1$ calibration samples that were taken at the estimated building.

To estimate the most probable floor, one selects the N1 $(N 1 \leq L 2)$ most similar calibration samples (filtering step A3), from those obtained in the previous step and computes the most probable floor (majority rule).

To estimate the most probable room, one selects the $M 1$ most similar calibration samples(filtering step A4), from within those obtained in the previous step and computes the most probable room (majority rule). Finally to estimate the geometric position, we take the geographic coordinates associated with the $P 1$ most similar calibration samples (filtering step A5) and compute the centroid of the associated positions.

\section{REAL WORLD EXPERIMENTS}

The positioning system discussed in previous sections was implemented and tested in a real set of buildings. In this section we describe the construction of the radio map and the test data used to evaluate the system performance. The data for the calibration and operational phases was collected using a laptop computer equipped with three similar network interface adapters (USB models), one network interface for each height.

The samples for the radio map were collected in two buildings at the University of Minho, each one of them with three floors. The test samples and calibration samples were collected in different days. A total of 392 calibration points were selected, three for each room. Within each room, the three calibration points were selected near the center of the room. At each calibration points, several samples were taken at each one of the three heights, totaling 9358 calibration samples. A total of 101 different AP were detected during this process.

For emulating the operational phase, 472 points were selected, uniformly distributed throughout the space of the two buildings. For each point, three samples were collected with an interval of around one second.

\section{RESULTS}

This section describes the results obtained through the use of the approach described in sections II and III, with the data described in the previous section.

\section{A. Filtering and Computational Load}

The filtering the Radio Map using the Test Sample as a key aims to reduce the computational load by reducing the number of samples for which the similarity has to be computed. Fig. 5 shows the reduction achieved on the size of the radio map. From results shown in Fig. 5 it can be seen that the filtered radio map contains only a small part of the initial radio map. Before the filtering process, the radio map has 9358 calibration samples. In the worst case scenario, after the filtering process the filtered radio map contains only 1403 calibration samples, which corresponds to a reduction of about $85 \%$. On average, the reduction on the size of the radio map is of $90 \%$, with the corresponding reduction of the time required to compute the similarity values.

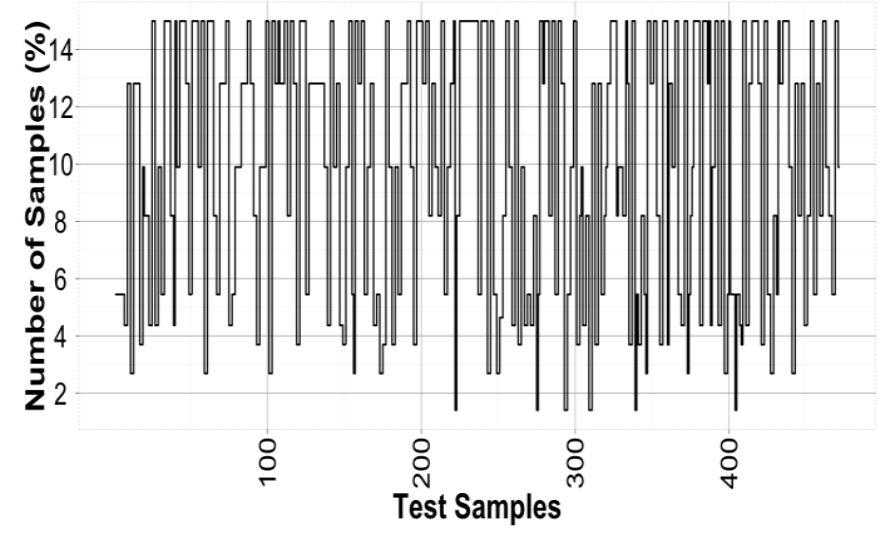

Figure 5. Impact of filtering on radio map size.

Fig. 6 shows the processing time for each of the 472 Test Samples. The average time to process each test sample is 5.1 milliseconds. Without the filtering of the radio map, the processing time would be around 120 milliseconds.

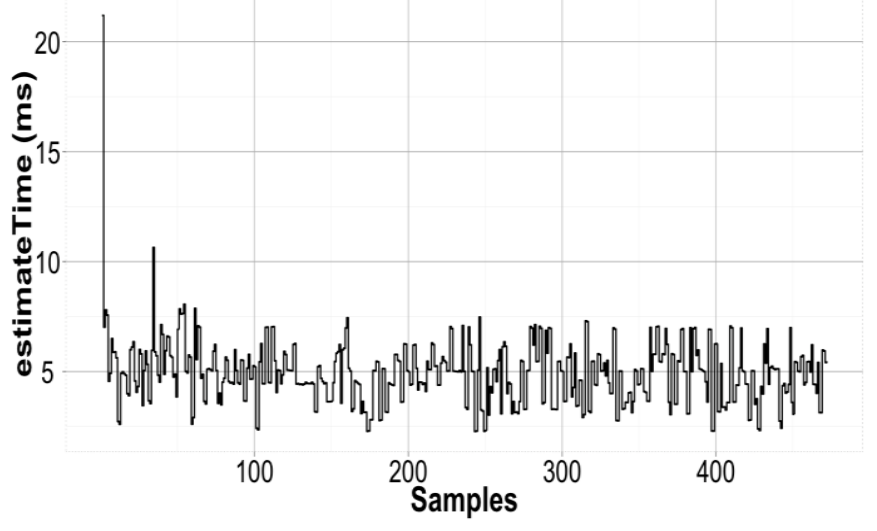

Figure 6. Processing time per test sample (Manhathan distance).

\section{B. Similarity Functions}

The choice of a similarity function has a significant impact on the accuracy of the positioning system. Table I lists some results obtained using the similarity functions described in section III-B. Six metrics are used to characterize the performance of each similarity function:

- $\quad$ Right Building Rate (RBR): refers to the frequency in correctly estimating the building where the test sample was taken;

- Right Floor Rate (RFR): refers to the frequency in correctly estimating the floor;

- $\quad$ Right Room Rate (RRR): refers to the frequency in correctly estimating the room;

- Average Error: refers to the average geometric error, in meters, as the average of the Euclidean distance between the correct position and the estimated position; 
- Maximum Error: refers to the maximum value of the geometric error, in meters;

- $\quad$ Processing Time: refers to the average time required to estimate the position.

TABLE I. RESUlts USING DifFERENT SiMILARITY FUnCTIONS $(\alpha=6$; $\beta=0)$

\begin{tabular}{ccccccc}
\hline $\begin{array}{c}\text { Similarity } \\
\text { Function }\end{array}$ & $\begin{array}{c}\boldsymbol{R B R} \\
\text { (Building) }\end{array}$ & $\begin{array}{c}\boldsymbol{R F R} \\
(\text { Floor })\end{array}$ & $\begin{array}{c}\boldsymbol{R R R} \\
(\mathrm{Room})\end{array}$ & $\begin{array}{c}\text { Avr. } \\
\text { Error } \\
(\mathrm{m})\end{array}$ & $\begin{array}{c}\text { Max. } \\
\text { Error } \\
(\mathrm{m})\end{array}$ & $\begin{array}{c}\text { Proc. } \\
\text { Time per } \\
\text { Sample } \\
(\mathrm{ms})\end{array}$ \\
\hline Manhattan & 1.0 & 0.995 & 0.741 & 3.351 & 13.763 & 5.1 \\
Euclidean & 1.0 & 0.993 & 0.677 & 3.745 & 14.901 & 6.6 \\
Tanimoto & 1.0 & 0.978 & 0.491 & 5.338 & 23.775 & 5.2 \\
\hline
\end{tabular}

In general, the Manhattan function can achieve better accuracy than the two other functions.

The values in Table I were obtained for $\alpha=6$ and $\beta=0$ (2). Fig. 7 shows how the error is affected by the values of these weighting parameters $\alpha$ and $\beta$. In this figure, the error for each one of the test samples is plotted. Positive values of the error refer to cases where the estimation process correctly estimated the building, floor and room. Therefore, positive errors are always distances within the same room. Negative values of the error refer to cases where the estimation is in the wrong room, and are just the additive inverse of the distance between the correct position and the estimated position.

These results show that the values chosen for the weighting parameters $\alpha$ and $\beta$ have a significant impact on the accuracy of estimation. Based on our experiments we found that $\alpha=6$ and $\beta=0$ provides a good accuracy for the Manhattan similarity function.

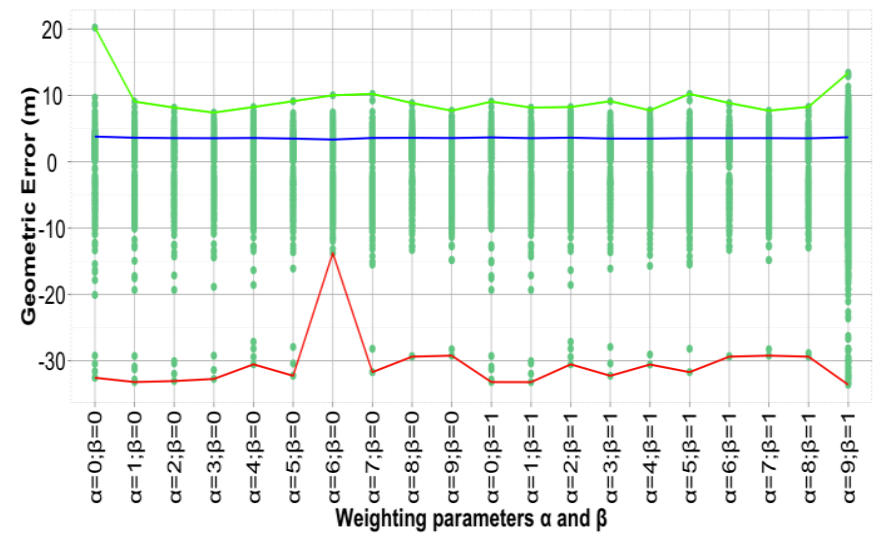

Figure 7. Performance of the proposed algorithm with different weighting parameters $\alpha$ and $\beta$ (Manhattan function).

\section{Filtering and Majority Rules}

Once the similarity with each calibration sample is computed, estimating the building, the floor, and the room is just a matter of applying a set of filters and majority rules, as explained in section III-C. At each filtering stage, a maximum number of samples are selected from within the samples obtained in the previous step. Four parameters control these maximum numbers of samples: L1, N1, M1 and P1, at filtering stages A2, A3, A4 and A5, respectively.

For our real world case, we observed that by adjusting the values of these parameters affects the accuracy of the positioning estimation. Initially, we investigated the impact of the number of Calibration Samples used on the estimation of the building (L1). We found that for values between 50 and 300 , the building is always estimated correctly $(\mathrm{RBR}=100 \%)$.

However, the larger the number of Calibration Samples, the longest is the time required to estimate the position. This is the reason why $\mathrm{L} 1=100$ was used. The optimum value for the L1 parameter also depends on the number and relative position of the calibrated buildings. The buildings used our experiments are relatively far from each other (around 100 meters apart) so the value of L1 has no significant impact on the RBR. For closer buildings, we expect to observe higher values for the optimum value of L1.

Estimating the floor is affected by the parameter N1. Fig. 8 shows how the RFR metric is affected by the value of N1. It can be seen that for $30 \leq \mathrm{N} 1 \leq 70$, the best performance is achieved for this scenario, with the floor being correctly estimated in more than $99 \%$ of the used Test Samples. Since a smaller number of samples leads to a shorter processing time, $\mathrm{N}=30$ was used as the optimum value.

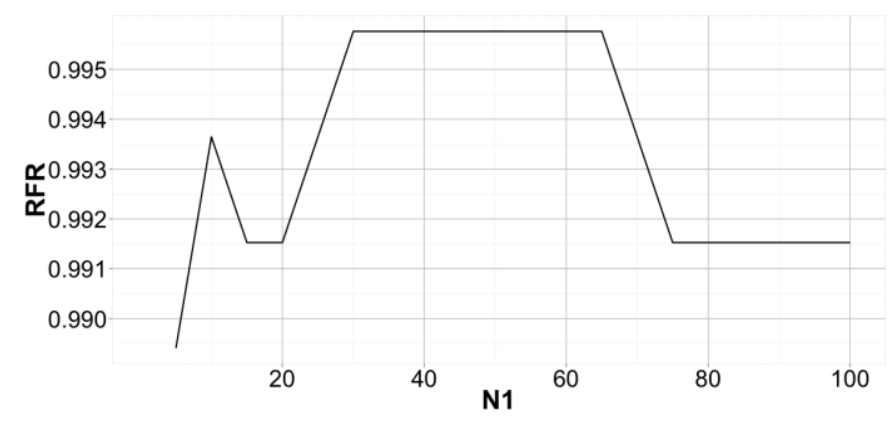

Figure 8. Impact of N1 parameter (majority rule) on right floor rate of the positioning system.

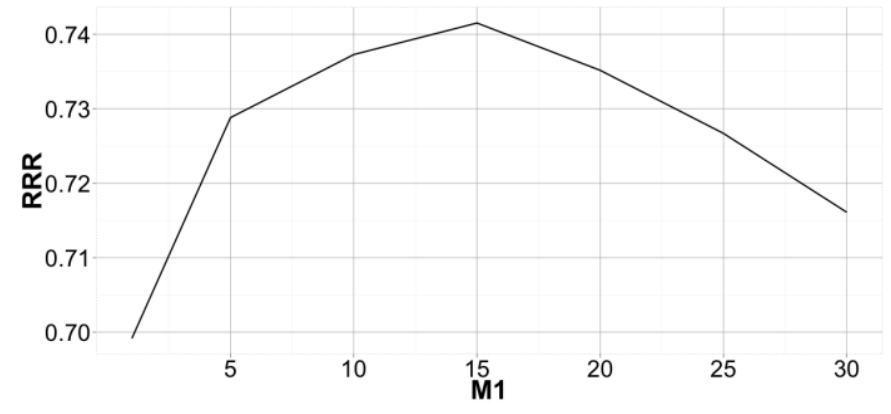

Figure 9. Impact of M1 parameter (majority rule) on the right room rate of the positioning system. 


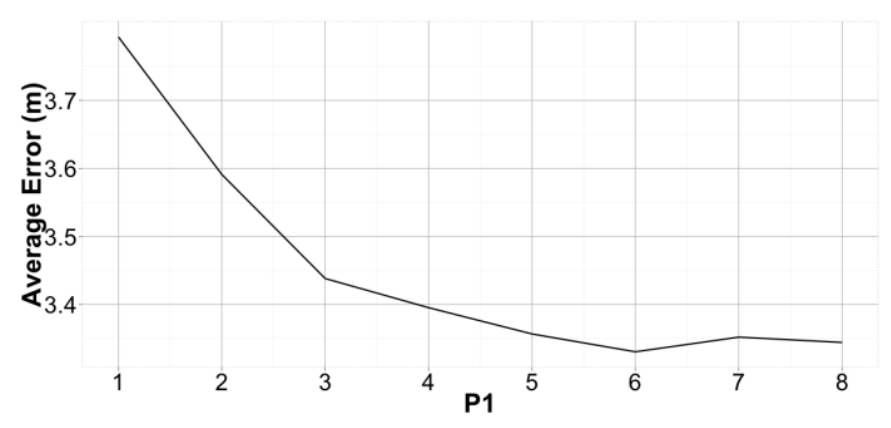

Figure 10. Impact of P1 parameter (majority rule) on average of the absolute error of the positioning system.

The experimental results for the value of M1 are illustrated in Fig. 9. As shown, the positioning algorithm is more accurate when M1 is between 15 and 20. Other values degrade considerably the system performance. Therefore, a value of M1=15 has been chosen as the optimum value.

Fig. 10 shows the impact of the value of $\mathrm{P} 1$ on the average error. Values of 6 or 7 are shown to provide the best results. The value of 7 was chosen as the optimum value.

\section{Overall Performance}

Fig. 11 shows the global results of positioning errors for the two calibrated buildings. Here, positive values of the error also means an error within the correct room, while negative values refer to the distance between the correct and estimated positions but with the estimated position within a wrong room. The cases where the estimated position is on a wrong floor or building are not shown (these cases are less that $0.5 \%$ of all cases, as shown in Table II). For easy of reading, the error values were sorted in increasing order of their absolute value.

These results show that most of the estimates are on the correct room and with an error shorter than 5 meters. There are, however, a few cases where the error is up to 10 meters. On the other hand, around one quarter of the estimates failed to pinpoint the correct room. Note, however, that in about half of these cases the absolute value of the error is shorter than 5 meters. These are the cases where the positioning estimate is on a neighbor room. In some cases, the estimated position is just across the wall separating the two rooms.

Table II shows the other metrics used to evaluate the performance of the positioning system. It shows that the positioning system is very successfully in estimating the correct building and floor. The performance in estimating the correct room still needs to be improved.

The results in Fig. 11 and Table II also show that using only the average error to characterize the performance of a positioning system might be misleading. Aspects such as the maximum error and the performance on estimating the correct room are not visible when using the average error only. The type of plot used in Fig. 11 provides a much richer view of the system performance. Note that the point at which the curve crosses the origin separates the samples where the room was correctly estimated (to the right) from those where the room was not correctly estimated (to the left).

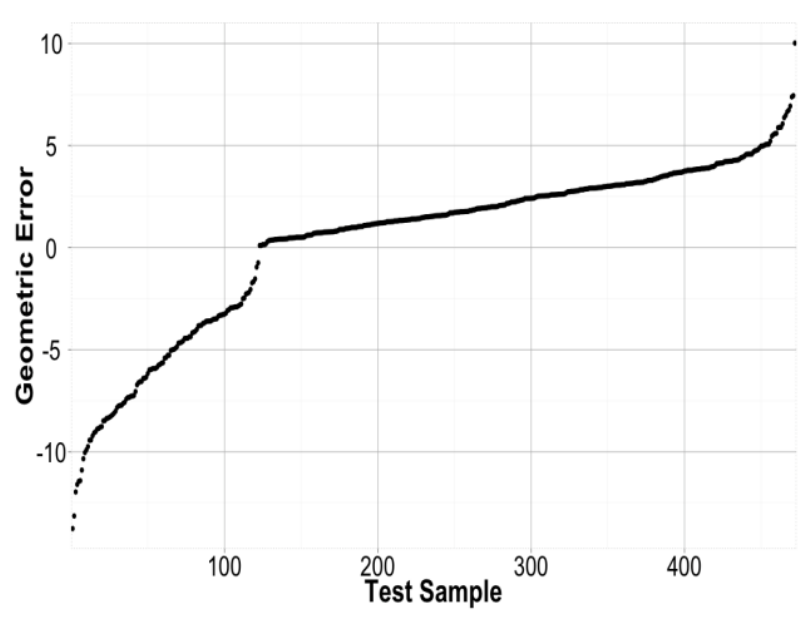

Figure 11. Overall performal of the proposed positioning system $(\alpha=6, \beta=$ $0, \mathrm{~L} 1=100, \mathrm{~N} 1=30, \mathrm{M} 1=15, \mathrm{P} 1=7$ ).

TABLE II. OVERALL RESUlts $(\alpha=6, \beta=0, \mathrm{~L} 1=100, \mathrm{~N} 1=30, \mathrm{M} 1=15$, $\mathrm{P} 1=7)$

\begin{tabular}{ccccc}
\hline $\begin{array}{c}\boldsymbol{R B R} \\
\text { (Building) }\end{array}$ & $\begin{array}{c}\boldsymbol{R F R} \\
(\text { Floor })\end{array}$ & $\begin{array}{c}\text { RRR } \\
(\text { Room })\end{array}$ & $\begin{array}{c}\text { Avr. } \\
\text { Error }\end{array}$ & $\begin{array}{c}\text { Max. } \\
\text { Error }(\mathrm{m})\end{array}$ \\
\hline 1.0 & 0.995 & 0.741 & 3.351 & 13.763 \\
\hline
\end{tabular}

\section{CONCLUSIONS}

We have described an algorithm for indoor position where the computational effort has been taken into account. The main advantage of this algorithm is that its performance is very acceptable for applications with real-time requirements.

The proposed method starts by estimating the building where the user is located, then determine the floor, the room and, finally, the most probable geometric position within the room. The results demonstrate that our strategy is feasible with an acceptable level of accuracy for many applications, such as locating people or equipment inside buildings, and at low computational effort.

Whether the estimation of the right building and floor can be performed before computing the similarity between the Test Sample and the Calibration Samples is being further investigated. This approach would contribute to reduce even more the computation effort. On the other hand, in cases of very close buildings, the right detection rate might be degraded.

The impact of different similarity functions used in our algorithm was investigated. Manhattan distance seems to perform better than the other two implemented functions. Also, the impact of the parameters used in the filtering and majority rules was evaluated. From the results it is clear that our algorithm is affected by the number of filtered samples in the various stages of the positioning algorithm. The impact of the adopted strategy in the computational load was also investigated. 
In addition, detailed statistics about the errors are provided, showing that the average metric error, often used by many authors, hides many aspects on the system performance. Resorting to diagrams like those illustrated in Fig.11 provides a much richer picture of a positioning system.

\section{ACKNOWLEDGMENT}

This work was supported by the FEDER program through the COMPETE and the Portuguese Science and Technology Foundation (FCT), within the context of projects SUM Sensing and Understanding human Motion dynamics (PTDC/EIA-EIA/113933/2009) and TICE.Mobilidade (COMPETE 13843).

\section{REFERENCES}

[1] A. Baniukevic, D. Sabonis, C. S. Jensen, and H. Lu, "Improving Wi-Fi Based Indoor Positioning Using Bluetooth Add-Ons," in Mobile Data Management (MDM), 2011 12th IEEE International Conference on, 2011, vol. 1, pp. $246-255$.

[2] K. Kaemarungsi and P. Krishnamurthy, "Properties of indoor received signal strength for WLAN location fingerprinting," in Mobile and Ubiquitous Systems: Networking and Services, 2004. MOBIQUITOUS 2004. The First Annual International Conference on, 2004, pp. $14-23$.
[3] C.-Y. Shih, L.-H. Chen, G.-H. Chen, E. H.-K. Wu, and M.-H. Jin "Intelligent radio map management for future WLAN indoor location fingerprinting," in Wireless Communications and Networking Conference (WCNC), 2012 IEEE, 2012, pp. 2769 -2773.

[4] S.-H. Fang, T.-N. Lin, and K.-C. Lee, "A Novel Algorithm for Multipath Fingerprinting in Indoor WLAN Environments," Wireless Communications, IEEE Transactions on, vol. 7, no. 9, pp. $3579-3588$, Sep. 2008.

[5] J.-G. Park, D. Curtis, S. Teller, and J. Ledlie, "Implications of device diversity for organic localization," in INFOCOM, 2011 Proceedings IEEE, 2011, pp. $3182-3190$.

[6] G. Lui, T. Gallagher, B. Li, A. G. Dempster, and C. Rizos, "Differences in RSSI readings made by different Wi-Fi chipsets: A limitation of WLAN localization," in Localization and GNSS (ICL-GNSS), 2011 International Conference on, 2011, pp. 53 -57.

[7] A. Arya, P. Godlewski, and P. Melle? and, "A Hierarchical Clustering Technique for Radio Map Compression in Location Fingerprinting Systems," in Vehicular Technology Conference (VTC 2010-Spring), 2010 IEEE 71st, 2010, pp. 1 -5.

[8] A. Arya, P. Godlewski, M. Campedel, and G. du C. ne, "Radio Database Compression for Accurate Energy-Efficient Localization in Fingerprinting Systems," IEEE Transactions on Knowledge and Data Engineering, vol. 99, no. PrePrints, 2011. 